\title{
A Correlation between Partial Discharge Characteristics and Dendrite Growth Stages in Polyethylene Insulation
}

\author{
M. M. Rezinkina, O. L. Rezinkin, and M. I. Nosenko \\ Kharkov Polytechnical Institute, Kharkov, Ukraine \\ e-mail:marinaR@kpi.kharkov.ua \\ Received May 15, 2000
}

(C) 2001 MAIK "Nauka/Interperiodica”.

Polyethylene is widely used as an insulating material (especially as cable insulation); therefore, of interest are ionization aging processes that cause its breakdown. For cable diagnostics, the partial discharge (PD) technique is used. In particular, PD characteristics may help in identifying the type of a defect [1]. Theoretical and experimental studies where the stage of dendrite growth in polyethylene was judged from the sequence of PD occurrence phases according to an applied voltage were summarized in [2]. It was concluded that this sequence is strictly determinate for either half-wave of the voltage when dendrites start to grow. Practical experience shows, however, that these processes are of stochastic nature and PD phases can hardly be reproduced regularly, as exemplified by our measurements.

Having detected PDs in a cable, one should assess how much dangerous dendrites responsible for their appearance are, i.e., how quickly they propagate. In our experiments, we tried to trace a correlation between dendrite growth stages and the characteristics of attendant PDs.

As was noted [3], the level of apparent PD charges during dendrite growth remains nearly the same, within $10^{-11}-10^{-12} \mathrm{C}$. The phases of PD occurrence rather than the PD charge level change significantly during treeing [12]. Technically, the detection of the PD initial stage without measuring the amount of apparent charge and sweeping PD current (relative to an applied voltage) seems to be the simplest and cheapest practical way of diagnostics. In our work, the initial phase of PDs vs. applied voltage was detected with an ADC and a PC.

In most laboratory studies of these processes, a needle-plane electrode system is applied (see, e.g., [3-6]). Such experimental geometry allows the location of a dendrite; moreover, it eliminates the latent time of its initiation, so that dendrite evolution can be observed from the very beginning.
Experiments were performed with braid-free pieces of an RK-100-7-13 cable. They were hot-deformed to measure $15 \times 15 \times 5 \mathrm{~mm}$. A needle with a tip radius of $2 \mu \mathrm{m}$ that served as a high-voltage electrode was arranged normally to the cable core at a distance of $1.5 \mathrm{~mm}$. The core was grounded, and the mains-frequency voltage applied to the needle was $U=7$ and 9 $\mathrm{kV}$. To avoid a corona and gap bridging over the surface, as well as to provide optical detection of a dendrite, the specimens were immersed in a transparent cell filled with transformer oil.

Tentative experiments showed that for this electrode system, the value $U=7 \mathrm{kV}$ was close to that of PD initiation. At this voltage, a dendrite stops propagating lengthwise 25-30 min after initiation; then, its branches thicken and simultaneously widen. The value $U=9 \mathrm{kV}$ is close to the breakdown voltage of the system, and the primary branch of a dendrite rapidly grew in all specimens. At this voltage, one can trace changes in the statistical distributions over PD initiation phases for dendrites growing lengthwise.

Usually, statistical characteristics of PDs are compared using the third and fourth central moments [1]: skewness, $\mathrm{S}^{+}$and $\mathrm{S}^{-}$, and kurtosis, $\mathrm{K}^{+}$and $\mathrm{K}^{-}$, which are measured for the positive and negative half-waves of an applied voltage, respectively. Statistical phase distributions of PDs that have been obtained thus far are, as a rule, multimodular; therefore, skewness and kurtosis derived directly from experimental data do not reflect the average distribution, which smooths out statistical spikes. Statistical phase distributions of PD that are constructed relative to the applied voltage are complex functions varying in accordance with the dendrite evolution stage. Hence, the most general approach to smooth them is the use of polynomials. In order to describe the phase distributions in terms of skewness and kurtosis, we approximated them by polynomials of the tenth to twentieth degree. Along with PD detection, we optically observed dendrites causing them. Typical 


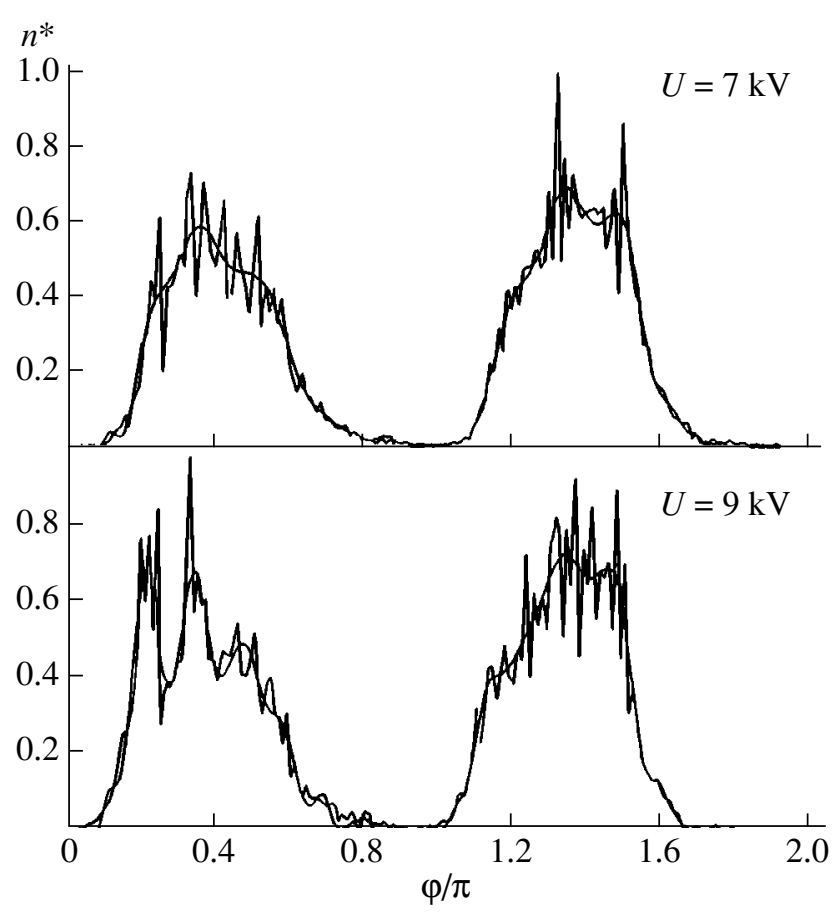

Fig. 1. Relative number of PDs in the polyethylene insulation $n^{*}=n / n_{\max }$ vs. phase $\varphi$ of their occurrence at the early stage of dendrite growth ( $n$ and $n_{\max }$ are the number of PDs and the maximum number of PDs, respectively).

statistical curves that describe the relative number of PDs vs. phase of their occurrence at $U=7$ and $9 \mathrm{kV}$ are shown in Figs. 1 (initial stage of dendrite growth) and 2 (later stage of dendrite growth). In Fig. 2, the curve taken for $U=9 \mathrm{kV}$ reflects the situation immediately before the breakdown of the gap, while that for $7 \mathrm{kV}$ reflects the situation $1.5 \mathrm{~h}$ after the initiation of a dendrite, when it stopped growing lengthwise. Both figures represent the distributions approximated (smoothed) by the twentieth-degree polynomial.

The phase distribution of PD initiation significantly varies in time and may characterize a dendrite evolution stage and the rate of propagation of its primary branch. At the initial stage, these statistical distributions have a similar shape, irrespective of the applied voltage: they are skewed both in the positive and in the negative halfwaves of the voltage. In the positive half-wave, they are positively skewed, their averages having $\mathrm{S}^{+}=0.278$ for $9 \mathrm{kV}$ and 0.372 for $7 \mathrm{kV}$. In the negative half-wave, the distributions are skewed weakly negatively, having $\mathrm{S}^{-}=$ -0.09 and -0.145 for 9 and $7 \mathrm{kV}$, respectively. Their average kurtosis is negative both in the positive and in the negative half-waves: $\mathrm{K}^{+}=-0.607, \mathrm{~K}^{-}=-0.753$ for $9 \mathrm{kV}$ and $\mathrm{K}^{+}=-0.409, \mathrm{~K}^{-}=-0.628$ for $7 \mathrm{kV}$. At the later stage of dendrite evolution, the distributions, as well as the associated skewness and kurtosis, change in a different way, depending on whether the dendrite contin-

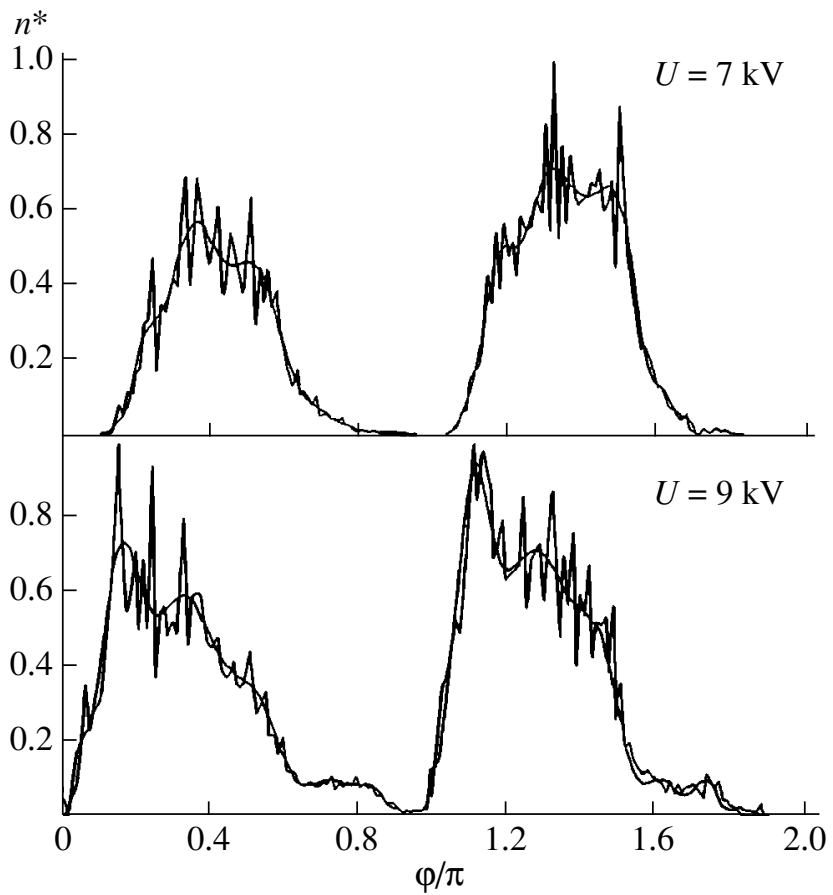

Fig. 2. $n^{*}$ vs. $\varphi 12 \mathrm{~min}$ after the initiation of a dendrite, when it still does not bridge the gap $(U=9 \mathrm{kV})$, and $1.5 \mathrm{~h}$ after the initiation of a dendrite, when it stops to grow lengthwise $(U=7 \mathrm{kV})$.

ues to propagate or not. If the primary branch of the dendrite grows, the characteristics after the breakdown of the gap $(U=9 \mathrm{kV})$ change: they remain similar in both half-waves, namely, negatively skewed $\left(\mathrm{S}^{+}=-\right.$ 0.558 and $\left.\mathrm{S}^{-}=-0.371\right)$, but become sharper $\left(\mathrm{K}^{+}=-0.19\right.$ and $\left.\mathrm{K}^{-}=-0.388\right)$. If the dendrite does not grow and only thickens $(U=7 \mathrm{kV})$, the distributions in both halfwaves become strongly skewed: $\mathrm{S}^{+}=-0.225, \mathrm{~S}^{-}=$ $-0.029, \mathrm{~K}^{+}=-0.407$, and $\mathrm{K}^{-}=-0.739$.

\section{CONCLUSIONS}

(1) At the initial stage of dendrite evolution and also when it stops growing lengthwise, the phase statistic distributions have the following features. They are smooth and skewed in both the positive and the negative half-waves of the applied voltage. In the former half-wave, they are positively skewed, while in the latter, they are nearly symmetric or negatively skewed (Figs. 1, 2; $U=7 \mathrm{kV}$ ).

(2) When the dendrite rapidly grows lengthwise and becomes dangerous (that is, can bridge the interelectrode gap), the phase distributions of PDs become similar in both half-waves: they are positively skewed and smoothed (Fig. 2, $U=9 \mathrm{kV}$ ). 


\section{REFERENCES}

1. F. H. Kreuger, E. Gulski, and A. Krivda, IEEE Trans. Electr. Insul. 28, 917 (1993).

2. M. Hoof and R. Patch, in Proceedings of the 9th International Symposium on High Voltage Engineering, 1995, Vol. 5, p. 5606.

3. G. S. Kuchinskiǔ, Partial Discharges in High-Voltage Constructions (Énergiya, Leningrad, 1979).
4. G. Krause, in Proceedings of the 7th International Symposium on High Voltage Engineering, Dresden, 1991, Vol. 7, p. 107.

5. H. Kawamura and M. Nawata, in Proceedings of the 10th International Symposium on High Voltage Engineering, Montreal, 1997.

6. G. Sagle, in Proceedings of the 10th International Symposium on High Voltage Engineering, Montreal, 1997.

Translated by V. Isaakyan 